\title{
Treatment of murine visceral leishmaniasis using an 8-hydroxyquinoline-containing polymeric micelle system
}

\author{
Mariana Costa Duarte ${ }^{\mathrm{a}, \mathrm{b}, 1}$, Letícia Martins dos Reis Lage ${ }^{\mathrm{a}, 1}$, Daniela Pagliara Lage ${ }^{\mathrm{b}}$, Vívian Tamietti Martins ${ }^{\mathrm{c}}$, \\ Ana Maria Ravena Severino Carvalho a , Bruno Mendes Roatt ${ }^{\mathrm{a}}$, Daniel Menezes-Souza ${ }^{\mathrm{a}, \mathrm{b}}$, \\ Carlos Alberto Pereira Tavares ${ }^{\mathrm{c}}$, Ricardo José Alves ${ }^{\mathrm{d}}$, \\ José Mário Barichello e,f , Eduardo Antonio Ferraz Coelho ${ }^{\mathrm{a}, \mathrm{b}, *}$ \\ a Departamento de Patologia Clínica, COLTEC, Universidade Federal de Minas Gerais, Belo Horizonte 31270-901, Minas Gerais, Brazil

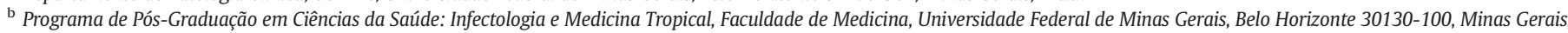 \\ Brazil \\ c Departamento de Bioquímica e Imunologia, Instituto de Ciências Biológicas, Universidade Federal de Minas Gerais, Belo Horizonte 31270-901, Minas Gerais, Brazil \\ ' Departamento de Produtos Farmacêuticos, Faculdade de Farmácia, Universidade Federal de Minas Gerais, Belo Horizonte 31270-901, Minas Gerais, Brazil \\ e Departamento de Farmácia, Escola de Farmácia, Universidade Federal de Ouro Preto, Ouro Preto 35400-000, Minas Gerais, Brazil

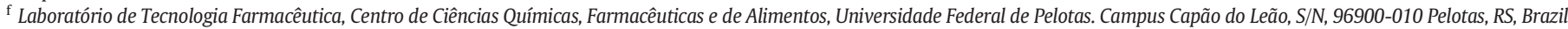

\section{A R T I C L E I N F O}

\section{Article history:}

Received 14 March 2016

Received in revised form 8 July 2016

Accepted 13 July 2016

Available online 15 July 2016

\section{Keywords:}

Visceral leishmaniasis

8-Hydroxyquinoline

Treatment

Toxicity

Poloxamer P407

Leishmania infantum

\begin{abstract}
A B S T R A C T
New therapeutics are urgently needed to treat visceral leishmaniasis $(\mathrm{VL})$. Due to the fact that drug discovery is a long and expensive process, the development of delivery systems to carry old and toxic drugs could be considered, as well as the evaluation of new molecules that have already shown to present biological activity. In this context, the present study evaluated the in vitro and in vivo antileishmanial activity of an 8-hydroxyquinoline (8-HQN)-containing polymeric micelle (8-HQN/M) system against Leishmania infantum, the main causative agent of VL in the Americas. The experimental strategy used was based on the evaluation of the parasite load by a limiting-dilution technique in the spleen, liver, bone marrow and draining lymph nodes of the infected and treated animals, as well as by a quantitative PCR ( $q \mathrm{PCR}$ ) technique to also assess the splenic parasite load. The immune response developed was evaluated by the production of IFN- $\gamma$, IL-4, IL-10, IL-12 and GM-CSF cytokines, as well as by antileishmanial nitrite dosage and antibodies production. Hepatic and renal enzymes were also investigated to verify cellular injury as a result of treatments toxicity. In the results, 8-HQN/M-treated mice, when compared to the other groups: saline, free amphotericin B (AmpB, as a drug control), 8-HQN and $\mathrm{B}-8-\mathrm{HQN} / \mathrm{M}$ (as a micelle control) showed more significant reductions in their parasite burden in all evaluated organs. These animals also showed an antileishmanial Th1 immunity, which was represented by high levels of IFN- $\gamma$, IL-12, GM-CSF and nitrite, associated with a low production of IL-4 and IL-10 and anti-Leishmania IgG1 isotype antibodies. In addition, any hepatic or renal damage was found in these treated animals. In conclusion, 8-HQN/M was effective in treating $L$. infantum-infected BALB/c mice, and can be considered alone, or combined with other drugs, as an alternative treatment for $\mathrm{VL}$.
\end{abstract}

(c) 2016 Elsevier Ireland Ltd. All rights reserved.

\section{Introduction}

Visceral leishmaniasis (VL) is an important disease worldwide leading to nearly 50,000 deaths annually [1-3]. The disease has gained greater importance in HIV co-infected patients as an opportunistic infection in areas where both infections are endemic [4]. In Brazil, VL is

\footnotetext{
* Corresponding author at: Laboratório do Programa de Pós-Graduação em Ciências da Saúde: Infectologia e Medicina Tropical, Faculdade de Medicina, Universidade Federal de Minas Gerais, 30130-100 Belo Horizonte, Minas Gerais, Brazil.

E-mail address: eduardoferrazcoelho@yahoo.com.br (E.A.F. Coelho).

1 These authors contributed equally to this work.
}

caused by Leishmania infantum species, where dogs are the main domestic reservoirs of the parasites and play an important role in the transmission cycle between humans and sandflies $[5,6]$. Symptomatic VL is characterized by clinical manifestations such as irregular fever, weight loss, pallor, splenomegaly, pancytopenia and it also carries a high risk of mortality in the absence of an adequate treatment $[7,8]$.

Chemotherapy based on the administration of pentavalent antimonials has been used to treat the disease [9]. Although these products are considered effective [10], there are evidences that their efficacy depends on the Leishmania species, geographic region, presence of resistant strains, therapeutic schemes and other factors [11-13]. In addition, the high incidence of side effects associated with toxicity in 
the liver, kidneys and spleen of the patients contributes to limit their use in clinical practice $[14,15]$. Other drugs have been also used to treat VL, although they also present problems related to their side effects and/or high cost [16]. Since the drug discovery is a very long and expensive process, an alternative way could be based on the development of technologies to improve the drugs delivery for treating this disease [17-19]. In addition to reducing the toxicity of conventional drugs, these systems can also diminish the number of doses administered to the patients, due to a slower liberation of the therapeutics, as well as exert a synergistic effect with the pharmaceutics employed [20]. In this context, studies for the development of polymeric micelle as delivery systems have been developed, based on the physicochemical and immune stimulatory properties of these products, which make them suitable to be used as drug delivery [21].

8-hydroxyquinoline (8-HQN) and its derivates have been experimentally evaluated in different studies as chemotherapeutic agents $[22,23]$, presenting antimicrobial activity $[24,25]$ and associated in the treatment of neurodegenerative diseases, such as Alzheimer's and Parkinson's disease [26]. In leishmaniasis, previous studies have shown that 8-HQN inhibited the growth of $L$. panamensis, $L$. tropica and $L$. major promastigotes $[27,28]$. In addition, in a recent study developed by our group, the in vitro antileishmanial activity of 8-HQN against stationary promastigotes and intra-macrophage amastigotes of $L$. amazonensis, L. braziliensis and L. infantum species was shown, and it was associated with a low toxicity in murine macrophages and in human red cells [29].

In this context, the purpose of the present study was to evaluate an 8-HQN-containing polymeric micelle (8-HQN/M) system developed to treat VL. BALB/C mice were infected with L. infantum stationary promastigotes and the parasite load in different organs was evaluated using the limiting dilution and quantitative PCR ( $q$ PCR) techniques. In addition, the immune response developed in the infected and treated animals was also assessed, determining the cytokine production by a capture ELISA and by flow cytometry; as well as investigating the nitrite production and humoral response in these animals. In addition, the treatments toxicity was evaluated by the measurement of renal and hepatic damage markers. Clinical symptoms and signals were also assessed.

\section{Materials and METHODS}

\subsection{Preparation of the $8-H Q N$-incorporated polymeric micelle and $A m p B$}

The 8-HQN-containing polymeric micelle system was prepared as described [30]. Briefly, P407 poloxamer $(18 \% \mathrm{w} / \mathrm{w})$ was diluted in a phosphate buffer $\mathrm{pH} 7.4$ (PBS $1 \times$ ) under moderate magnetic agitation for $18 \mathrm{~h}$ at $4{ }^{\circ} \mathrm{C}$. Then, $8 \mathrm{mg}$ of 8 -HQN (ACS reagent $99 \%$, catalog 252565, Sigma-Aldrich, St. Louis, MO, USA) were added to a new tube containing $500 \mu \mathrm{L}$ of dichloromethane PA, and solubilized using vortex. The solution was immediately added to the prepared $\mathrm{P} 407$ poloxamer, under vigorous magnetic agitation and in an ice bath, until a viscous emulsion was obtained. The dichloromethane was evaporated by rotary evaporation (Buchi, Flawil, Switzerland), and the final concentration of the formulation $(8 \mathrm{mg} / \mathrm{mL})$ was adjusted using purified water. This product is a transparent yellow gel, at room temperature. Empty micelle $(18 \% \mathrm{w} / \mathrm{w})$ were prepared using the same protocol, but without adding 8 -HQN. All products were maintained at room temperature, until their use. Free AmpB ( $1 \mathrm{mg}$; Cristália, São Paulo, São Paulo, Brazil) was resuspended in $2 \mathrm{~mL}$ of a methanol/DMSO $(9: 1 \mathrm{v} / \mathrm{v})$ solution, and this preparation was maintained at $-80^{\circ} \mathrm{C}$, until use.

\subsection{Animals}

This study was approved by the Committee on the Ethical Handling of Research Animals (CEUA) from UFMG, Belo Horizonte, Minas Gerais, Brazil, with the protocol number 182/2012. Female BALB/c mice
(8 weeks age) were obtained from the breeding facilities of the Department of Biochemistry and Immunology, Institute of Biological Sciences (ICB), Federal University of Minas Gerais (UFMG); and were maintained under specific pathogen-free conditions.

\subsection{Parasites}

L. infantum (MHOM/BR/1970/BH46) strain was used. Parasites were grown in complete Schneider's medium (Sigma-Aldrich), which was composed by Schneider's medium plus $20 \%$ heat-inactivated fetal bovine serum (FBS, Sigma-Aldrich), $20 \mathrm{mM}$ L-glutamine, $200 \mathrm{U} / \mathrm{mL}$ penicillin, $100 \mu \mathrm{g} / \mathrm{mL}$ streptomycin and $50 \mu \mathrm{g} / \mathrm{mL}$ gentamicin, $\mathrm{pH} 7.4$ at $24^{\circ} \mathrm{C}$. The soluble L. infantum antigenic (SLA) extract was prepared as described previously [31]. The amastigote-like forms were prepared following a technical protocol described by [32] and modified by [33]. Briefly, $1 \times 10^{9}$ stationary-phase promastigotes were washed in sterile PBS $1 \times$. Then, parasites were incubated in the presence of $5 \mathrm{~mL}$ of FBS, for $48 \mathrm{~h}$ at $37^{\circ} \mathrm{C}$. After, they were washed two times in sterile PBS $1 \times$ and visualized in an optical light microscopy. The cellular density was estimated by counting in a Neubauer chamber, and their morphology was evaluated after staining by Giemsa [34].

\subsection{In vitro antileishmanial activity}

The inhibition of the Leishmania growth was assessed in vitro by cultivating L. infantum stationary promastigotes $\left(1 \times 10^{6}\right.$ cells $)$ in the presence of serial dilutions of 8-HQN/M or B-8-HQN/M (700, 350, 175, 87.5, $43.75,21.88,10.93,5.47,2.74,1.37$ and $0.68 \mu \mathrm{M}$ ) in 96 -well culture plates (Nunc, Nunclon ${ }^{\circledR}$, Roskilde, Denmark), for 48 h at $24{ }^{\circ} \mathrm{C}$. AmpB $(10.8,5.4,2.7,1.35,0.68,0.34,0.17,0.08$ and $0.04 \mu \mathrm{M})$ was used as control. Cell viability was assessed by MTT method, according previously described [34]. The $50 \%$ inhibitory concentration $\left(\mathrm{IC}_{50}\right)$ of the compounds was determined by applying the sigmoidal regression of the concentration-response curves. Data shown are representative of three different experiments, performed in triplicate, which presented similar results.

\subsection{Cytotoxicity assay}

The cytotoxicity was evaluated in murine macrophages and in human red cells. For this, murine macrophages were collected from the peritoneal cavities of BALB/c mice elicited with $3 \mathrm{~mL}$ of thioglycolate, which was administered five days before the experiments. Then, cells $\left(5 \times 10^{5}\right.$ per well $)$ were incubated in the presence of serial dilutions of $8-\mathrm{HQN} / \mathrm{M}$ or B-8-HQN/M (700, 350, 175, 87.5, $43.75,21.88,10.93,5.47,2.74,1.37$ and $0.68 \mu \mathrm{M})$ in 96 -well plates (Nunc), for $48 \mathrm{~h}$ at $37^{\circ} \mathrm{C}$. The inhibition of $50 \%$ of the macrophage viability $\left(\mathrm{CC}_{50}\right)$ was assessed by cleavage of $2 \mathrm{mg} / \mathrm{mL}$ of MTT. AmpB $(10.8,5.4$, $2.7,1.35,0.68,0.34,0.17,0.08$ and $0.04 \mu \mathrm{M})$ was used as control. The selectivity index (SI) was determined by calculating the ratio between the $\mathrm{CC}_{50}$ and $\mathrm{IC}_{50}$ values. The hemolytic activity was investigated by incubating serial dilutions of 8-HQN/M or B-8-HQN/M (700, 350, 175, 87.5, $43.75,21.88,10.93,5.47,2.74,1.37$ and $0.68 \mu \mathrm{M}$ ) with a $5 \%$ human red blood cell suspension, for $1 \mathrm{~h}$ at $37^{\circ} \mathrm{C}$, according a technical protocol described [34]. The compounds concentration needed to cause $50 \%$ of hemolysis in human red cells $\left(\mathrm{RBC}_{50}\right)$ was determined by applying a sigmoidal regression using the concentration-response curves. Data shown are representative of three independent experiments, performed in triplicate, which presented similar results.

\subsection{In vivo infection and treatment}

BALB/c mice ( $\mathrm{n}=12$ per group) were subcutaneously infected into the right hind footpad with $1 \times 10^{7}$ stationary promastigotes of $\mathrm{L}$. infantum. Forty-five days after infection, animals were treated daily, from day 0 to day 15 after the first administration of the dose, by 
subcutaneous injections into their left hind footpad, as described: (a) control group: mice received PBS $1 \times$; (b) Amphotericin B (AmpB) group: mice received $20 \mu \mathrm{g}$ of free AmpB (equal to $1 \mathrm{mg} / \mathrm{Kg}$ body weight); (c) 8-HQN group: mice received $100 \mu \mathrm{g}$ of free 8-HQN (equal to $5 \mathrm{mg} / \mathrm{Kg}$ body weight); (d) empty micelle group (B-8-HQN/M): mice received $100 \mu \mathrm{g}$ of micelle (equal to $5 \mathrm{mg} / \mathrm{Kg}$ body weight); (e) 8-HQN micelle group (8-HQN/M): mice received $100 \mu \mathrm{g}$ of 8-HQN/M (equal to $5 \mathrm{mg} / \mathrm{Kg}$ body weight). The concentrations of free $\mathrm{AmpB}$, free 8-HQN, 8-HQN/M and B-8-HQN/M used to treat the animals were obtained after a previous dose-response experiment, in which the maximum concentrations of the compounds able to effectively treat the infected animals, without causing toxic effects, were defined (data not shown). One-day after the end of treatments, animals were euthanized and the parasite load in the spleen, liver, bone marrow (BM) and draining lymph nodes ( $\mathrm{dLN}$ ) were evaluated by limiting dilution and qPCR (only spleen) techniques. Data shown in this study are representative of two independent experiments, which presented similar results.

\subsection{In vivo toxicity}

Serum samples of the infected and treated animals were collected one-day after the end of treatments, in order to perform the biochemical analysis. For this, the hepatic function was analyzed by dosage of aspartate aminotransferase (AST) and alanine aminotransferase (ALT), whereas the nephrotoxicity was evaluated by examining the levels of blood urea nitrogen and serum creatinine. All biochemical assays were performed using commercial kits (Labtest Diagnostica ${ }^{\circledR}$, Belo Horizonte, Minas Gerais, Brazil), according to manufacturer's instructions. In addition, the individual animal weights were measured before, during and after the treatments, and results obtained in the treated groups were compared to those derived from the control, to evaluate the drugs toxicity.

\subsection{Parasite burden}

\subsubsection{Limiting dilution technique}

A limiting-dilution technique developed by [35] and modified by [36] was used. For this, spleen, liver, BM and dLN were collected, weighed and homogenized using a glass tissue grinder in sterile saline. Log-fold serial dilutions were performed in Schneider's medium with a $10^{-1}$ to $10^{-12}$ dilution, and each sample was plated in triplicate and read 7 days after the beginning of the culture at $24^{\circ} \mathrm{C}$. Results were expressed as the negative log of the titer (i.e., the dilution corresponding to the last positive well), which was adjusted per milligram of organ.

\subsection{2. qPCR analysis}

The parasite load in the spleen of the infected and treated animals was also evaluated by a qPCR technique. For this, spleen DNA was extracted using a phenol-chloroform method [37], and it was amplified using the forward (CCTATTTTACACCAACCCCCAGT) and reverse (GGGTAGGGGCGTTCTGCGAAA) oligonucleotides. The technical procedure was developed on StepOne ${ }^{\mathrm{TM}}$ Instrument (48 wells-plate; Life Technologies) using $2 \times$ SYBR $囚$ Green PCR Master Mix ( $5 \mu \mathrm{L}$; Applied Biosystems), with $2 \mathrm{mM}$ of each primer $(1 \mu \mathrm{L})$ and $4 \mu \mathrm{L}$ of DNA ( $5 \mathrm{ng} /$ $\mu \mathrm{L})$. The samples were incubated at $95^{\circ} \mathrm{C}$ for $10 \mathrm{~min}$, and then submitted to 40 cycles of $95^{\circ} \mathrm{C}$ for $15 \mathrm{~s}$ and $60^{\circ} \mathrm{C}$ for $1 \mathrm{~min}$, and during each time, fluorescence data were collected. Results were converted into number of parasites per nucleated cells (multiplied by one thousand to facilitate the analysis of results).

\subsection{Evaluation of the immune response}

\subsubsection{Capture ELISA}

The cytokine profile was evaluated in the splenocytes of the infected and treated animals, one-day after the end of treatments, as described
[38]. For this, cell suspensions $\left(5 \times 10^{6}\right)$ were plated in 24-well plates (Nunc) and incubated in complete DMEM (unstimulated, background control), which was composed by DMEM plus 20\% FBS, 20 mM L-glutamine, $100 \mathrm{U} / \mathrm{mL}$ penicillin, $50 \mu \mathrm{g} / \mathrm{mL}$ streptomycin, and $25 \mu \mathrm{g} / \mathrm{mL}$ gentamicin, at a pH 7.4, or stimulated with L. infantum SLA $(20 \mu \mathrm{g} / \mathrm{mL})$ for $48 \mathrm{~h}$ at $37{ }^{\circ} \mathrm{C}$ in $5 \% \mathrm{CO}_{2}$. IFN- $\gamma$, IL-4, IL-10, IL-12p70 and GM-CSF levels were assessed in the culture supernatants by a sandwich ELISA provided in commercial kits (BD OptEIA TM set mouse, Pharmingen, San Diego, CA, USA), following the manufacturer's instructions.

\subsubsection{Flow cytometry}

IFN- $\gamma$, TNF- $\alpha$ and IL-10 cytokines were evaluated in the spleen cells of the infected and animals using a flow cytometry assay. For this, splenocytes were incubated in the presence of $1 \mathrm{~mL}$ of complete RPMI 1640 medium in polypropylene tubes (Pharmingen). Cells were nonstimulated (background control) or stimulated with L. infantum SLA $(20 \mu \mathrm{g} / \mathrm{mL})$. Then, they were incubated with phorbol myristate acetate (PMA, $25 \mathrm{ng} / \mathrm{mL}$ ) or ionomycin $(1 \mu \mathrm{g} / \mathrm{mL})$, diluted in complete RPMI 1640 medium and cultures were incubated for $48 \mathrm{~h}$ at $37^{\circ} \mathrm{C}$ in $5 \% \mathrm{CO}_{2}$. After, the technical protocol was developed according previously described [39]. The frequency of $\mathrm{CD}^{+}$and $\mathrm{CD} 8^{+} \mathrm{T}$ cells, as well as the expression of intracytoplasmic cytokines were determined following an analysis based on their relative flow cytometry size (forward laser scatter - FSC) and granularity (side laser scatter - SSC) graphs. After the selection of the interest region R1 containing $\mathrm{FSC}^{\mathrm{Low}}$ and $\mathrm{SSC}^{\mathrm{Low}}$ phenotype cells, graphs of density plot distribution of CD4/FL1 or CD8/ FL1 versus IFN- $\gamma / F L 2$, TNF- $\alpha / F L 2$, or IL-10/FL2 were performed to determine the percentage of IFN- $\gamma^{+}, \mathrm{TNF}-\alpha^{+}$, and IL- $10^{+} \mathrm{T}$ cells in the cultured splenocytes. Results were expressed as indexes, which were determined by the ratio between the percentage of $\mathrm{CD} 4^{+}$and $\mathrm{CD} 8^{+} \mathrm{T}$ cells in the parasite-stimulated cultures, by the values obtained using the unstimulated cultures (SLA/CC ratio).

\subsection{Nitrite production and humoral response}

The nitrite production was evaluated in the culture supernatants that were used to quantify the cytokines, by Griess method [40]. The humoral response was evaluated by determining the antileishmanial IgG1 and IgG2a isotype antibodies levels, as described [41]. Briefly, $L$. infantum SLA was used as an antigen (1.0 $\mu$ g per well) and serum samples were diluted at 1:100 in PBS-T (PBS $1 \times$ plus Tween-20 0.05\%). The anti-mouse IgG1 and IgG2a horseradish-peroxidase conjugated antibodies (Sigma-Aldrich) were used both 1:10,000 diluted in PBS-T, and reactions were developed through incubation with $2 \mathrm{mg}$ ortophenylenediamine, $2 \mu \mathrm{L} \mathrm{H}_{2} \mathrm{O}_{2} 30 \mathrm{vol}$. and $10 \mathrm{~mL}$ citrate-phosphate buffer $\mathrm{pH} 5.0$, for $30 \mathrm{~min}$ in the dark; when they were stopped by adding $25 \mu \mathrm{L} \mathrm{H}_{2} \mathrm{SO}_{4} 2 \mathrm{~N}$. The optical density was read in an ELISA microplate spectrophotometer at $492 \mathrm{~nm}$.

\subsection{Statistical analysis}

In the statistical assays, the one-sample Kolmogorov-Smirnoff test was used to determine whether a variable was normally distributed, and the data showed a Gaussian distribution. Thus, the one-way analysis of variance (ANOVA) followed by Bonferroni's post-test were used for comparisons between groups. Results are showed as mean \pm standard deviation of the experimental groups. Differences were considered significant when $P<0.05$.

\section{Results}

\subsection{In vitro antileishmanial activity and cytotoxicity}

Initially, the products in vitro antileishmanial activity was evaluated against the stationary promastigotes and amastigotes-like stages of $L$. infantum. In the results, the $\mathrm{IC}_{50}$ values of $8-\mathrm{HQN} / \mathrm{M}, \mathrm{B}-8-\mathrm{HQN} / \mathrm{M}$ and 
free AmpB for the promastigotes were of $1.8 \pm 0.02,29.0 \pm 0.7$ and $1.19 \pm 0.04 \mu \mathrm{M}$, respectively, whereas the values for the amastigoteslike were of $2.1 \pm 0.1,34.4 \pm 2.2$ and $1.4 \pm 0.3 \mu \mathrm{M}$, respectively. The $\mathrm{CC}_{50}$ values of $8-\mathrm{HQN} / \mathrm{M}, \mathrm{B}-8-\mathrm{HQN} / \mathrm{M}$ and free AmpB indicating cytotoxicity in murine macrophages were of $126.0 \pm 3.5,217.0 \pm 3.4$ and $9.5 \pm$ $0.4 \mu \mathrm{M}$, respectively, whereas the SI calculated for each product was $70.0,7.5$ and 8.0, respectively, demonstrating that $8-\mathrm{HQN} / \mathrm{M}$ was the one that showed the highest SI value and, consequently, is safer to be used in mammalian hosts. As an additional toxicity parameter, the $\mathrm{RBC}_{50}$ values for $8-\mathrm{HQN} / \mathrm{M}, \mathrm{B}-8-\mathrm{HQN} / \mathrm{M}$ and free AmpB indicating hemolytic activity in human red cells were of $4.1 \pm 0.2,2.9 \pm 0.05$ and $0.01 \pm 0.003 \mu \mathrm{M}$, respectively.

\subsection{Evaluation of the in vivo toxicity}

Treatments using free AmpB, free 8-HQN, 8-HQN/M or B-8-HQN/ $\mathrm{M}$ were performed in the $L$. infantum-infected BALB/c mice. One-day after the end of the therapeutic regimens ( 60 days after beginning of experiments), serum samples of the animals were collected and hepatic and renal damage markers were evaluated. In the results, the animals of the AmpB group were the only ones presenting statistically significant increase in their levels of urea, creatinine, ALT, AST compared to the control groups treated with saline and empty micelle (B-8-HQN/M). The 8-HQN/M group presented the lowest levels of all measured enzymes (Fig. 1). Clinical symptoms such as ataxia, body weight loss and weakness were observed mainly in the saline (8 of 12 animals) and free AmpB (all animals) groups, since these animals showed negative variations of $8 \%$ and $15 \%$, respectively, in their body weight, possibly reflecting the disease evolution and/or drug toxicity. On the other hand, animals treated with 8-HQN/M showed a positive variation in their body weight, reaching a maximum of $4 \%$ compared with the beginning of the treatment. Mice that received free $8-\mathrm{HQN}$ or $\mathrm{B}-8-\mathrm{HQN} / \mathrm{M}$ presented a negative variation in their body weight of $5 \%$ and $3 \%$, respectively. In this context, the treatment using $8-\mathrm{HQN} / \mathrm{M}$ did not promote any renal or hepatic damage, and no clinical alteration was detected in these treated animals.
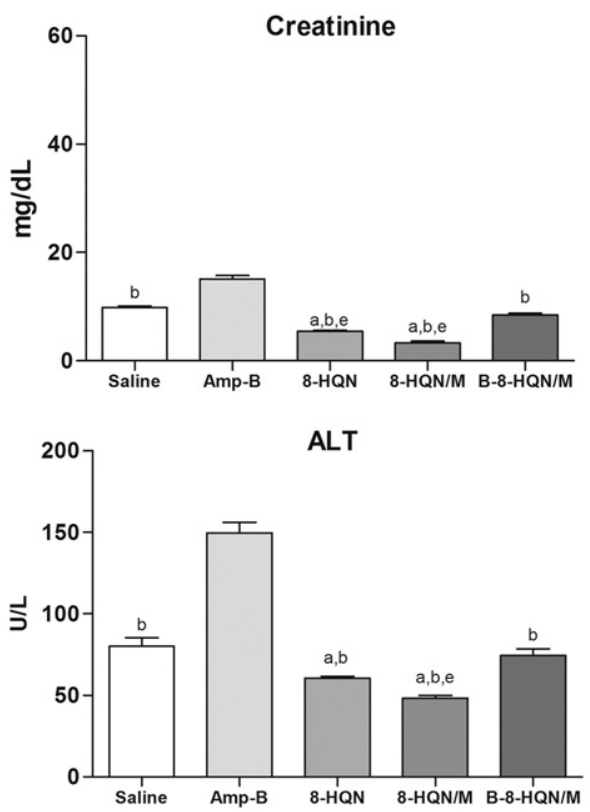

\subsection{In vivo treatment of $L$. Infantum-infected $B A L B / c$ mice}

Mice were infected with $L$. infantum stationary promastigotes and, 45 days after challenge, when the animals presented a chronic infection [42], they were treated during 15 days with free AmpB, 8-HQN, 8-HQN/ $\mathrm{M}$ or B-8-HQN/M. A previous kinetic study was performed to determine the most appropriated therapeutic schedule to be used in the infected animals (data not shown). Then, animals were treated once daily receiving subcutaneous injections with the different therapeutics and, one-day after the end of the treatments, they were euthanized and the parasite burden in the liver, spleen, BM and dLN was investigated by limiting dilution and $q \mathrm{PCR}$ (only spleen) techniques. Evaluating the limiting dilution results, the treatments using free AmpB, 8-HQN and 8$\mathrm{HQN} / \mathrm{M}$ were all effective in reducing the parasite load in the animals, when compared to the results found for the B-8-HQN/M and saline groups (Fig. 2). The 8-HQN/M group showed a more effective reduction in the animals parasite load, since the most significant parasitism reductions were found in the liver (1.2- and 1.5-log reductions), spleen (1.4and 1.7-log reductions), dLN (3.0- and 2.2-log reductions) and BM (1.9and 1.4-log reductions), when compared to the free AmpB and 8-HQN groups, respectively. These results were also found when the splenic parasite load was evaluated by $q$ PCR technique (Fig. 3).

\subsection{Immune response generated in the infected and treated animals}

To investigate the cytokine profile generated in the infected and treated animals, the IFN- $\gamma$, IL-4, IL-10, IL-12 and GM-CSF levels were measured in the supernatants of spleen cells cultured in vitro and stimulated with parasite antigens, during $48 \mathrm{~h}$ at $37{ }^{\circ} \mathrm{C}$ in $5 \% \mathrm{CO}_{2}$. This incubation time was based on previous technical protocols performed by our group, when therapeutics or vaccine candidates against leishmaniasis were studied $[34,36,38]$. As shown in Fig. 4, spleen cells from mice treated with 8-HQN/M were those that produced significantly higher levels of IFN- $\gamma$, IL-12 and GM-CSF than those produced by animals treated with free AmpB, 8-HQN or B-8-HQN/M; suggesting the development of a more polarized Th1 immunity in these animals. In addition, free AmpB and 8-HQN-treated mice also showed a higher production of these cytokines, when compared to the saline and B-8-HQN/M groups.
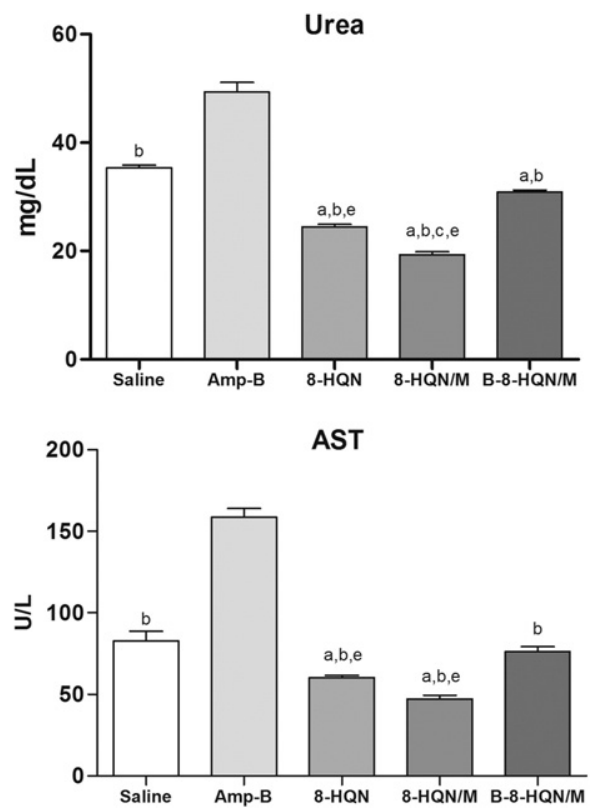

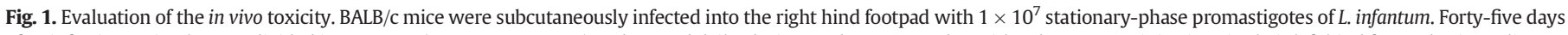

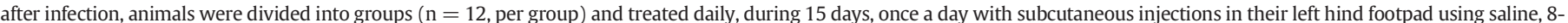

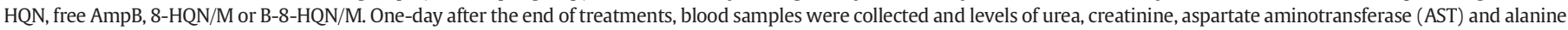

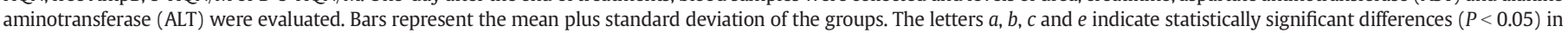
relation to the saline, free AmpB, 8-HQN and B-8-HQN/M groups, respectively. Experiments were repeated once and presented similar results. 

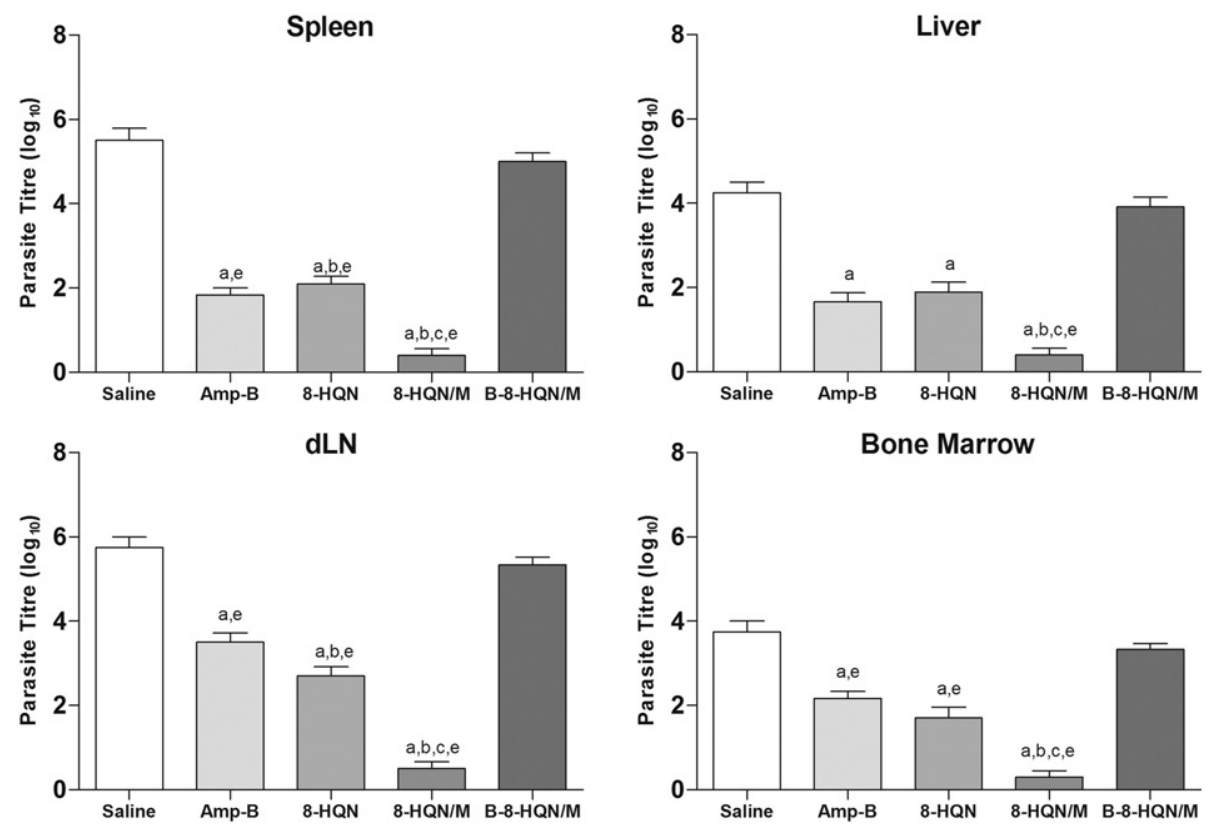

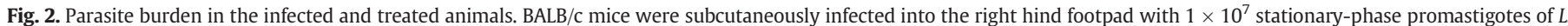

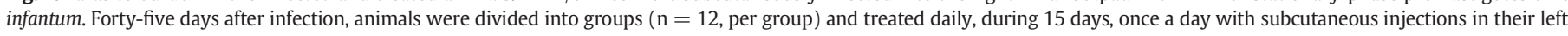

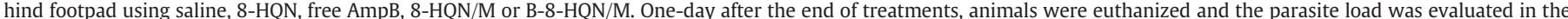

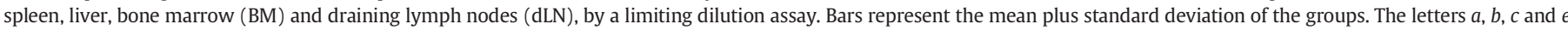

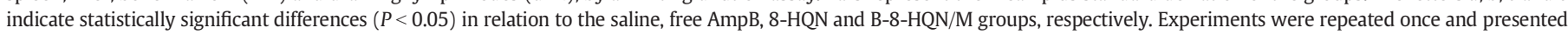
similar results.

On the other hand, IL- 4 and IL-10 levels were significantly higher in the saline, free AmpB and B-8-HQN/M groups; although animals from saline group presented the highest levels of these cytokines. In addition, free 8-HQN-treated animals also showed high levels of IL-4 and IL-10, when compared to the treatment using $8-\mathrm{HQN} / \mathrm{M}$. To characterize the effect of the treatments in the phenotypic profile and cytokine production in the spleen cells stimulated with SLA, a flow cytometry analysis was performed. In the results, the T cells phenotypic profile showed that $8-\mathrm{HQN} / \mathrm{M}$ lead to an increase in the IFN- $\gamma$ production by both T cells subtypes, being represented by populations of IFN- $\gamma$-producing $\mathrm{CD}^{+}$and $\mathrm{CD}^{+} \mathrm{T}$ cells (Fig. 5). On the other hand, these animals

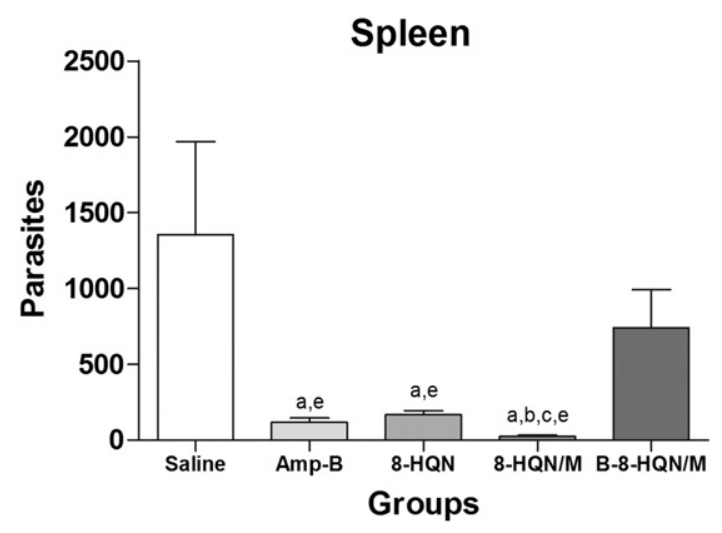

Fig. 3. Splenic parasite burden evaluated by a quantitative PCR (qPCR). BALB/c mice were subcutaneously infected into the right hind footpad with $1 \times 10^{7}$ stationary promastigotes of $L$. infantum. Forty-five days after infection, animals were divided into groups $(n=12$, per group) and treated daily, during 15 days, once a day with subcutaneous injections of saline, 8-HQN, free AmpB, 8-HQN/M or B-8-HQN/M. One-day after the end of treatments, animals were euthanized and the parasite load was also evaluated in their spleen by a qPCR assay. Results are expressed by the number of parasites per 1000 nucleated cells of the host. Bars represent the mean plus standard deviation of the groups. The letters $a, b, c$ and $e$ indicate statistically significant differences $(P<0.05)$ in relation to the saline, free AmpB, 8-HQN and B-8-HQN/M groups, respectively. Experiments were repeated once and presented similar results. showed lower levels of IL-10-producing T cells, when compared to the values found for the other groups. In addition, a significant increase of $\mathrm{CD}^{+}$and $\mathrm{CD}^{+}{ }^{+} \mathrm{T}$ cell indexes (stimulated cell/non-stimulated cell) producing IFN- $\gamma$ and TNF- $\alpha$, associated with a low production of IL10, were found in the 8-HQN/M group (Fig. 6).

Nitrite levels were evaluated as indicators of NO production. In the results, the treatments using free $\mathrm{AmpB}, 8-\mathrm{HQN}$ and $8-\mathrm{HQN} / \mathrm{M}$ induced a high nitrite production, when compared to the saline and B-8-HQN/M groups; however, the highest levels of this molecule were found in the 8-HQN/M-treated mice (Fig. 7), since spleen cells of the saline, free AmpB, 8-HQN, 8-HQN/M and B-8-HQN/M groups showed anti-Leishmania nitrite values of $0.75 \pm 0.13,1.45 \pm 0.22,4.73 \pm 0.51,8.31 \pm$ 0.83 and $1.92 \pm 0.38 \mu \mathrm{M}$, respectively. When the humoral response in

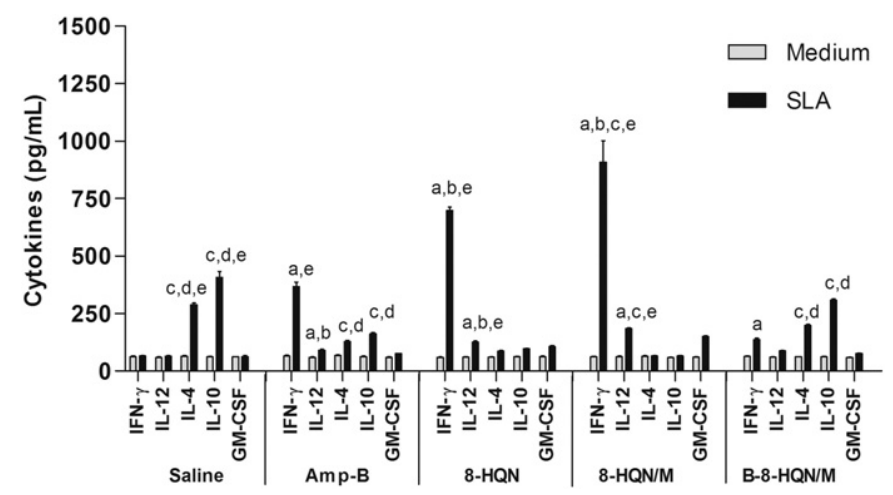

Fig. 4. Immune response in the infected and treated animals. Single cells suspensions were obtained from the spleen of infected animals, one-day after the end of treatments, and cells were unstimulated (medium, background control) or stimulated with $L$. infantum SLA $(20 \mu \mathrm{g} / \mathrm{mL})$, for $48 \mathrm{~h}$ at $37{ }^{\circ} \mathrm{C}$ in $5 \% \mathrm{CO}_{2}$. IFN- $\gamma$, IL-12, IL-4, IL-10 and GM-CSF levels were measured in culture supernatants by capture ELISA. Bars represent the mean plus standard deviation of the groups. The letters $a, b, c, d$ and $e$ indicate statistically significant differences $(P<0.05)$ in relation to the saline, free AmpB, 8-HQN, 8-HQN/M and B-8-HQN/M groups, respectively. Experiments were repeated once and presented similar results. 
CD4 ${ }^{+}$Cells

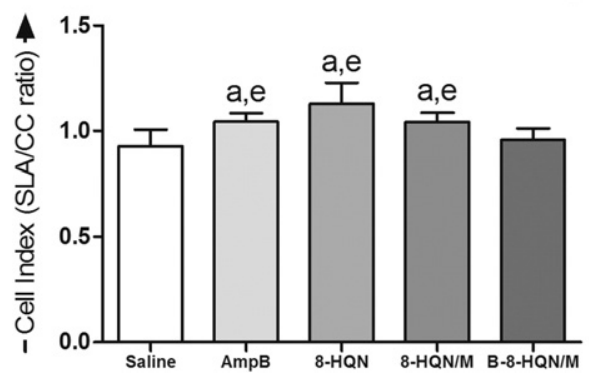

A

$\mathrm{CD}^{+}$Cells

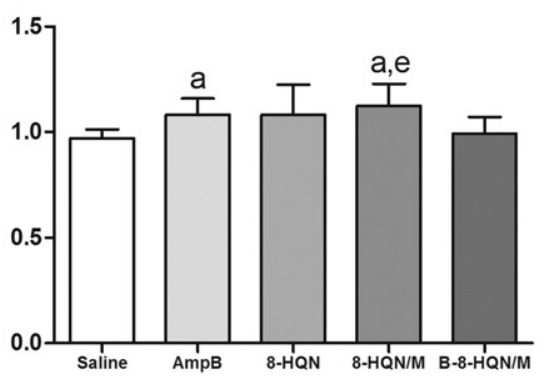

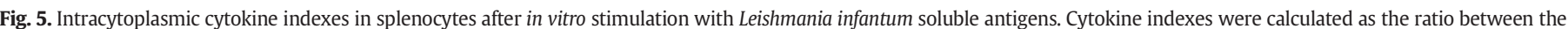

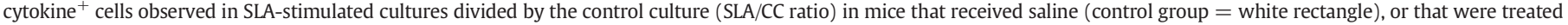

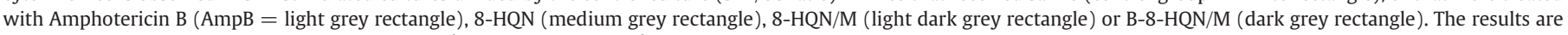

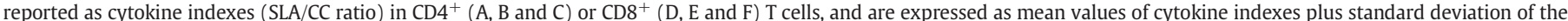

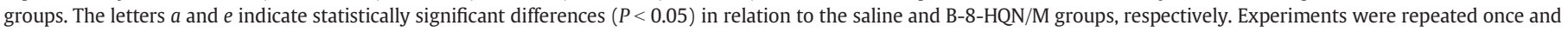
presented similar results.

CD4 ${ }^{+}$Cells

IFN- $\gamma$

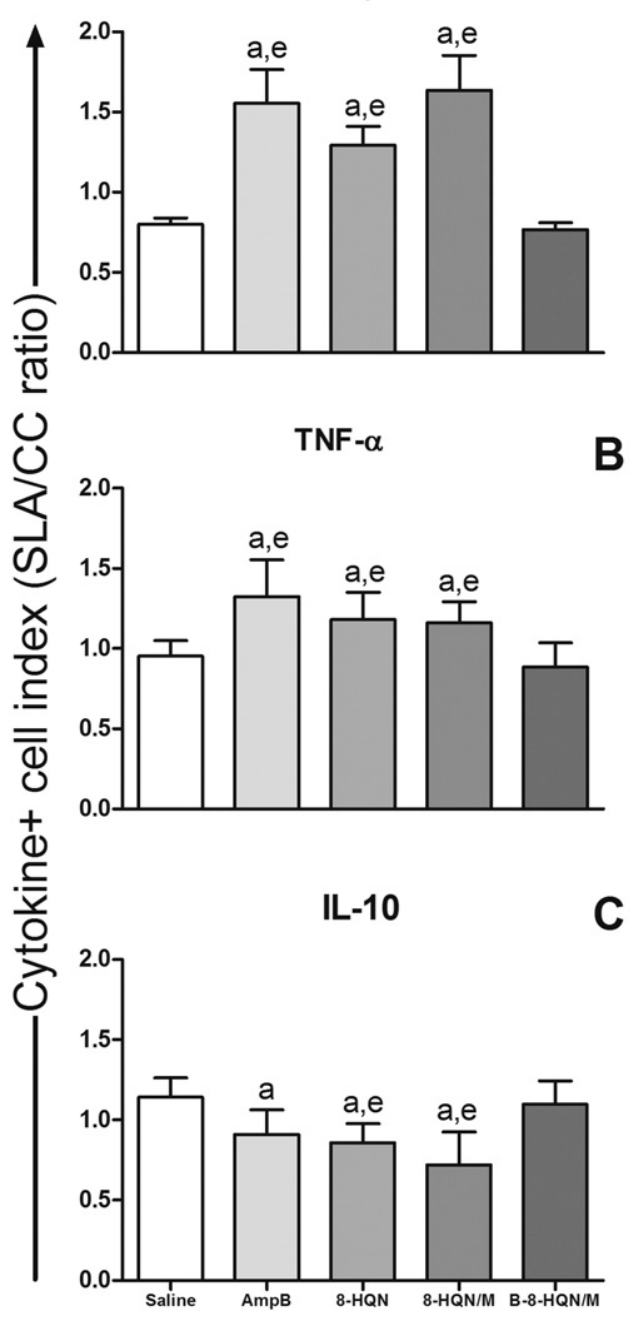

$\mathrm{CD}^{+}$Cells

A

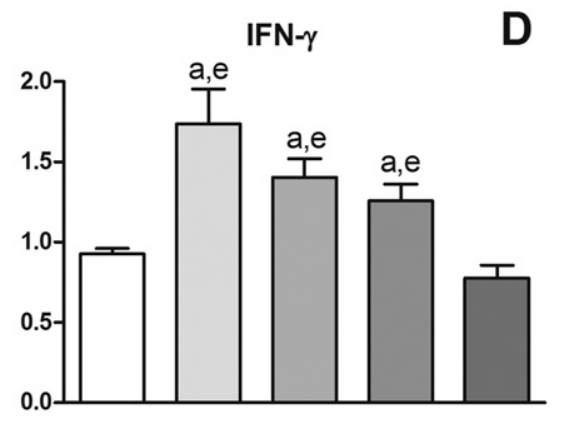

B

TNF- $\alpha$

E

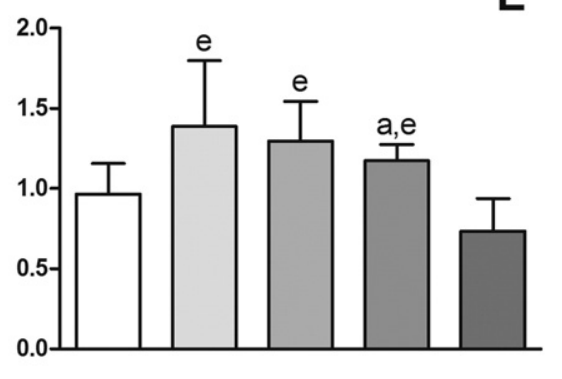

IL-10

F

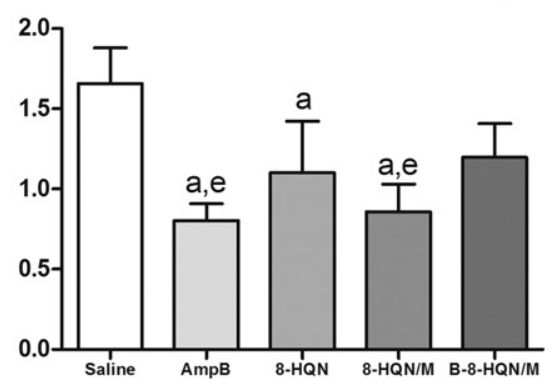

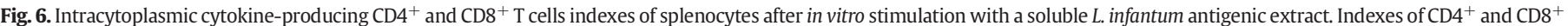

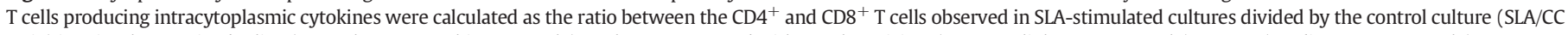

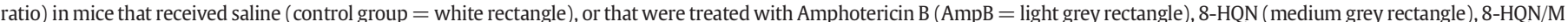

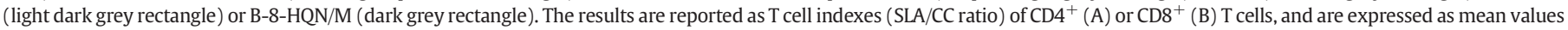

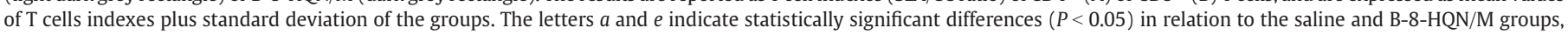
respectively. Experiments were repeated once and presented similar results. 


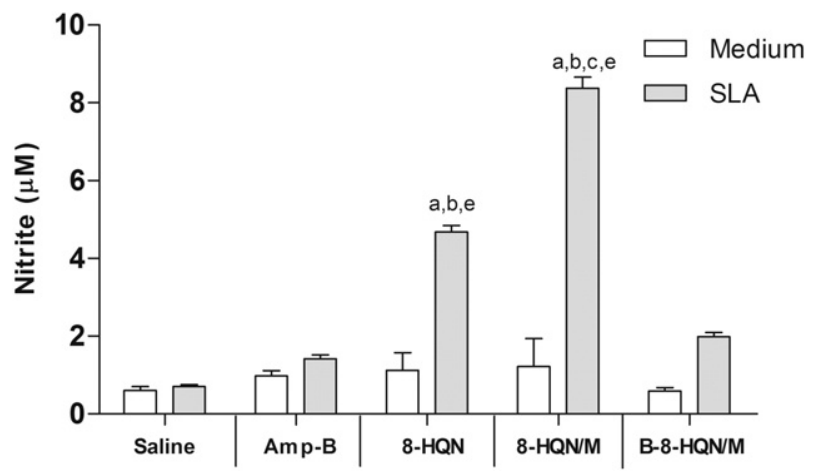

Fig. 7. Nitrite production. Single cells suspensions were obtained from the spleen of infected animals, one-day after the end of the treatments, and cells were unstimulated (medium, background control) or stimulated with L. infantum SLA $(20 \mu \mathrm{g} / \mathrm{mL})$, for $48 \mathrm{~h}$ at $37{ }^{\circ} \mathrm{C}$ in $5 \% \mathrm{CO}_{2}$. The presence of nitrite in the supernatants was analyzed by the Griess method. Bars represent the mean plus standard deviation of the groups. The letters $a, b, c$ and $e$ indicate statistically significant differences $(P<0.05)$ in relation to the saline, free AmpB, 8-HQN and B-8-HQN/M groups, respectively. Experiments were repeated once and presented similar results.

the infected and treated animals was evaluated, the treatment using 8$\mathrm{HQN} / \mathrm{M}$ induced higher levels of anti-Leishmania IgG2a isotype antibodies, when compared to the IgG1 levels, as well as a higher ratio between these isotypes, in comparison to the results obtained in the other groups (Fig. 8). Animals from the saline and B-8-HQN groups presented a predominance of antileishmanial IgG1 antibodies, whereas free AmpBtreated animals presented similar IgG1 and IgG2a isotypes levels.

\section{Discussion}

The treatment of VL has been considered unsatisfactory, since the available treatments are accompanied by the occurrence of side effects such as electrocardiographic alterations, ventricular tachyarrhythmias, laboratory abnormalities, nephrotoxicity and bone marrow suppression $[13,43]$. AmpB has been considered an effective drug against Leishmania, but it is also toxic for patients. Lipid-based formulations such as Ambisome ${ }^{\circledR}$, AmphocilH ${ }^{\circledR}$ and Abelcet ${ }^{\circledR}$ have been developed and, despite improvements in the AmpB therapeutic index, their use still remains limited, mainly due to their high cost $[44,45]$. In this context, the search for new antileishmanial drugs continues, as well as the search to obtain new delivery systems to carry them, presenting no toxic effects and low cost of formulations [44,46-49]. In this context, copolymers composed by hydrophilic and hydrophobic polymers have been developed [50]. These molecules present a size ranging from 10 to $100 \mathrm{~nm}$ and a high structural stability provided by the entanglement of polymer chains in the inner core. Amphiphilic block copolymers consisting of hydrophilic and hydrophobic segments have attracted attention because of their unique phase in aqueous media [51]. In particular, poloxamer 407 (P407; Pluronic ${ }^{\circ}$ F127, BASF, Germany), a thermoreversible copolymer with an amphiphilic nature consisting of a central hydrophobic block of polypropylene oxide (PPO) flanked by hydrophilic polyethylene blocks (PEG-PPO-PEG), has gained interest and presented effective results in the design of dermal, subcutaneous and transdermal delivery systems [52].

These poloxamers have been applied for a broad range in the biomedical and pharmaceutical industries [53]. The ease of production, stability at $4{ }^{\circ} \mathrm{C}$ and room temperature and the low cost of formulations are attractive conditions for their use [54]. For instance, experimental studies using Pluronic®-based polymeric micelle as anti-cancer agents [5557] and antileishmanial compounds [50] have been performed. Recently, a poloxamer 407-based paramomycin-containing micelle nanogel system was developed and its in vivo tolerance was assayed by transepidermal water loss and by ex vivo cytotoxicity in RAW and VERO cells; besides of its antileishmanial activity against Leishmania promastigotes. In the results, the authors showed that this product presented negligible toxicity and an effective antileishmanial activity against $L$. major and $L$. infantum promastigotes. They concluded that the formulation provided a controlled, effective and safe delivery of paramomycin for the animals [57]. In the present study, a molecule that was experimentally shown by our group to present an in vitro antileishmanial activity against $L$. infantum amastigotes and promastigotes [29], was used to construct a micelle system and this product was evaluated in the treatment of murine VL. To the best of our knowledge, this is the first time that a poloxamer 407-based system for 8-HQN delivery has been developed to treat against VL.

Mice are considered a known model for studies involving experimental chemotherapy, and there are many susceptible lineages for leishmaniasis [58]. Initially, studies are developed to evaluate the compounds in vitro antileishmanial activity $[29,59]$ and, after confirming their biological effectiveness, in vivo studies in murine models are developed to evaluate their therapeutic efficacy for the treatment of the disease [60-62]. In our study, BALB/c mice were infected with $1 \times 10^{7} \mathrm{~L}$. infantum stationary promastigotes and, 45 days after, they were treated by subcutaneous injections with different therapeutic regimens, during 15 days. After this time (60 days after the beginning of infection), animals were euthanized and parasitological and immunological parameters were evaluated by collecting serum samples and organs of the animals. We have chosen to evaluate these parameters one-day after the end of the treatments, since this mouse lineage, when infected by L. infantum, usually tends to present a hepatic parasite load peak between 20 and 30 days after infection, and between 45 and 60 days in the spleen and BM, when the parasite load tends to diminish and parasites are usually eliminated by the animals immune system [40,63-65].

In VL, the development of a Th1 immune response is related with the protection against infection $[65,66]$. In this context, therapeutics inducing the development of a Th1 response, based on high levels of IFN- $\gamma$ that will activate infected macrophages to kill the internalized parasites via NO production, could contribute more effectively to eliminate
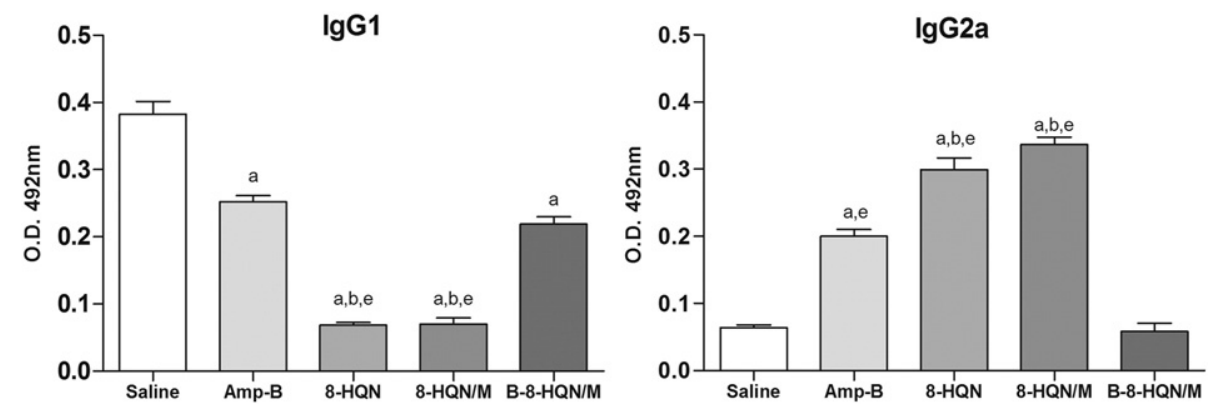

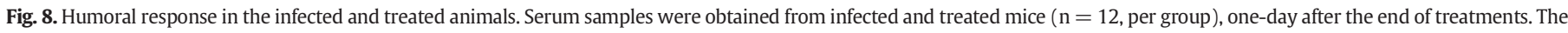

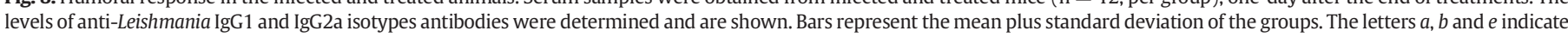
statistically significant differences $(P<0.05)$ in relation to the saline, free AmpB and B-8-HQN/M groups, respectively. Experiments were repeated once and presented similar results. 
Leishmania. However, other studies have shown that antileishmanial compounds are not able to induce the nitrite production, nor iNOS expression, suggesting that the leishmanicidal activity observed may be due to a mechanism different than the NO production by the infected macrophages. Valadares et al. [34] showed that the Agaricus blazei Murill mushroom was unable to induce the NO production by these phagocytic cells. Tempone et al. [67] showed that a snake venom presented an effective leishmanicidal activity but without inducing NO production. According to this data, it can be speculated that therapeutics can act either stimulating the parasitized cells to kill the parasites or by other mechanisms than NO production, such as the transposition of the macrophages membranes to reach the internalized parasites through a direct effect of these products [68].

In our study, the treatment using 8-HQN/M induced high levels of IFN- $\gamma$, IL-12 and GM-CSF in the spleen cells in vitro stimulated with parasite antigens, which were associated with a low production of IL-4 and IL-10, demonstrating the development of a polarized and antileishmanial Th1 immunity in these treated mice. These animals presented the lowest levels of parasitism in different organs, when two distinct technical procedures were adopted. Although significant reductions in the parasite load have been found in the animals that were treated with free AmpB or 8-HQN, when compared to the saline and B-8-HQN/M groups, the most significant reduction of the parasite load was obtained in the 8-HQN/M group permitting, at least to infer, the occurrence of an association between the Th1 immune response and the reduction of infection in these animals.

AmpB was used as a drug control in the present study. The selective activity of this product against different Leishmania species is due to its higher affinity for 24-substituted sterols such as ergosterol and episterol, which are abundant in the plasma membrane of these eukaryotic microorganisms, in comparison to the cholesterol found in the membrane of mammalian cells $[69,70]$. In our study, significant alterations in hepatocyte-associated serum enzymes, combined with significant increases in the levels of AST and ALT, were found in the animals that were treated with this drug. Also, results showed that blood urea nitrogen and creatinine were increased in the free AmpB-treated mice. In contrast, in the 8-HQN/M-treated mice, no enzymatic alteration was found. Therefore, it can be concluded that, although the free AmpB has reduced the parasitism in the infected animals, it was toxic to them. Also, although this drug has been used as a control, the application of a delivery system control should be considered, such as Ambisome ${ }^{\circledR}$, aiming to compare not only the efficacy and/or the toxicity of the free drug, but also the involvement of the delivery system used to treat the animals. As a consequence, the absence of this delivery control could be considered as a limitation of the present study.

8-hydroxyquinolines are heterocyclic compounds characterized by a moderate metal-binding affinity. The interest in this molecule and its derivates has grown exponentially in the last two decades, as they are privileged structures for the design of new drug candidates that exert a range of biological effects on different targets [71]. The experimental studies involving 8-HQN such as neuroprotection, anticancer, antibacterial and antifungal activities have been further promoted by the synthetic versatility of $8-\mathrm{HQN}$, which allows the generation of a large number of derivatives. The mechanism of action of this molecule has been based on its interaction with metal ions. On the complexation with copper, iron salts or cellular copper, 8-HQN inhibits the in vitro proliferation of cancer cells and in vivo tumor growth [71]. In the present study, when this molecule was associated with a delivery system based on polymeric micelle, the formulation was highly effective in treating against the disease, and a Th1 immune response was found in the treated animals. This response could be a direct effect of the treatment or an indirect action of the immune system, since when reducing the parasite load, the susceptibility profile could be diminished and the immune system of the animals could be activated to guarantee the development of a protective immunity.
Certainly, other therapeutic regimens based on the reduction in the number of doses or including other antileishmanial compounds could be considered. Nevertheless, we believe that the present model can be considered adequate, since this micelle formulation is cheap to produce, presents a good stability and can be administered into mammalian hosts by a subcutaneous route, whereas the other drugs are usually administered by intravenous or intramuscular injections and the treatment regimens take a long time.

\section{Conclusion}

Taking results together, after immunological and parasitological analysis, we conclude that the 8-HQN/M composition is highly effective in treating $L$. infantum-infected BALB/c mice, and can be considered alone, or combined with other drugs, as an alternative treatment for VL.

\section{Conflict of interest}

The authors confirm that they have no conflicts of interest in relation to this work.

\section{Acknowledgments}

This work was supported by grants from Instituto Nacional de Ciência e Tecnologia em Nano-biofarmacêutica (INCT-NanoBiofar), FAPEMIG (CBB-APQ-00819-12 and CBB-APQ-01778-2014), and CNPq (APQ-482976/2012-8, APQ-488237/2013-0, and APQ-467640/2014-9). EAFC is a grant recipient of CNPq.

\section{References}

[1] P.J. Guerin, P. Olliaro, S. Sundar, M. Boelaert, S.L. Croft, P. Desjeux, et al., Personal view visceral leishmaniasis: current status of control, diagnosis, and treatment, and a proposed research and development agenda personal view, Lancet 2 (2002) 494-501.

[2] F. Chappuis, S. Sundar, A. Hailu, H. Ghalib, S. Rijal, R.W. Peeling, et al., Visceral leishmaniasis: what are the needs for diagnosis, treatment and control? Nat. Rev. Microbiol. 5 (2007) 873-882.

[3] D.S. Marinho, C.N.P.R. Casas, C.C.D.A. Pereira, I.C. Leite, Health economic evaluations of visceral leishmaniasis treatments: a systematic review, PLoS Negl. Trop. Dis. 9 (2015), e0003527.

[4] J. Alvar, I.D. Vélez, C. Bern, M. Herrero, P. Desjeux, J. Cano, et al., Leishmaniasis worldwide and global estimates of its incidence, PLoS One 7 (2012), e35671.

[5] R. Lainson, J.J. Shaw, New world leishmaniasis, in: F.E.G. Cox, D. Wakelin, S.H. Gillespie, D.D. Despommier (Eds.), Topley \& Wilson's Microbiology and Microbial Infections: Parasitology, 10th ed.Hodder Arnold ASM Press, London 2005, pp. 313-349.

[6] M. Gramiccia, L. Gradoni, The current status of zoonotic leishmaniases and approaches to disease control, Int J. Parasitol. 35 (2005) 1169-1180.

[7] G. Michel, C. Pomares, B. Ferrua, P. Marty, Importance of worldwide asymptomatic carriers of Leishmania infantum (L. chagasi) in human, Acta Trop. 119 (2011) 69-75.

[8] H. Apa, I. Devrim, N. Bayram, R. Deveci, G. Demir-Özek, Ö.U. Carti, Liposomal amphotericin B versus pentavalent antimony salts for visceral Leishmania in children, Turk. J. Pediatr. 55 (2013) 378-383.

[9] S. Sundar, J. Chakravarty, Antimony toxicity, Int. J. Environ. Res. Public Health 7 (2010) 4267-4277.

[10] G. Wortmann, R.S. Miller, C. Oster, J. Jackson, N. Aronson, A randomized, doubleblind study of the efficacy of a 10- or 20-day course of sodium stibogluconate for treatment of cutaneous leishmaniasis in United States military personnel, Clin. Infect. Dis. 35 (2002) 261-267.

[11] G.A.S. Romero, M.V.F. Guerra, M.G. Paes, V.O. Macêdo, Comparison of cutaneous leishmaniasis due to Leishmania (Viannia) braziliensis and L. (V.) guyanensis in Brazil: therapeutic response to meglumine antimoniate, Am.J.Trop. Med. Hyg. 65 (2001) 456-465.

[12] S. Sundar, Drug resistance in Indian visceral leishmaniasis, Tropical Med. Int. Health 6 (2001) 849-854.

[13] S.L. Croft, G.H. Coombs, Leishmaniasis: current chemotherapy and recent advances in the search for novel drugs, Trends Parasitol. 19 (2003) 502-508.

[14] P.D. Marsden, Pentavalent antimonials: old drugs for new diseases, Rev. Soc. Bras. Med. Trop. 18 (1985) 187-198.

[15] M. Barral-Netto, A. Barral, C. Brodskyn, E.M. Carvalho, S.G. Reed, Cytotoxicity in human mucosal and cutaneous leishmaniasis, Parasite Immunol. 17 (1995) 21-28.

[16] S.K. Bhattacharya, T.K. Jha, S. Sundar, C.P. Thakur, J. Engel, H. Sindermann, et al., Efficacy and tolerability of miltefosine for childhood visceral leishmaniasis in India, Clin. Infect. Dis. 38 (2004) 217-221.

[17] M.S. Butler, Natural products to drugs: natural product derived compounds in clinical trials, Nat. Prod. Rep. 22 (2005) 162-195. 
[18] K.H. Bleicher, H.-J. Böhm, K. Müller, A.I. Alanine, Hit and lead generation: beyond high-throughput screening, Nat. Rev. Drug Discov. 2 (2003) 369-378.

[19] J.P. Hughes, S.S. Rees, S.B. Kalindjian, K.L. Philpott, Principles of early drug discovery, Br. J. Pharmacol. 162 (2011) 1239-1249.

[20] M.A. Chávez-Fumagalli, T.G. Ribeiro, R.O. Castilho, S.O. Fernandes, V.N. Cardoso, C.S. Coelho, et al., New delivery systems for amphotericin B applied to the improvement of leishmaniasis treatment, Rev. Soc. Bras. Med. Trop. 48 (2015) 235-242.

[21] S.R. Croy, G.S. Kwon, Polymeric micelle for drug delivery, Curr. Pharm. Des. 12 (36) (2006) 4669-4684

[22] P. Collery, F. Lechenault, A. Cazabat, E. Juvin, L. Khassanova, A. Evangelou, et al., Inhibitory effects of gallium chloride and tris(8-quinolinolato) gallium III on A549 human malignant cell line, Anticancer Res. 20 (2000) 955-958.

[23] C.C. Tzeng, K.H. Lee, T.C. Wang, C.H. Han, Y.L. Chen, Synthesis and cytotoxic evaluation of a series of gamma-substituted gamma-aryloxymethyl-alpha-methylenegamma-butyrolactones against cancer cells, Pharm. Res. 17 (2000) 715-719.

[24] D.L. Lentz, H. Gershon, H. Marini, New antifungal agents that inhibit the growth of Candida species: dichlorinated 8-quinolinols, Mycopathologia 147 (1999) 117-120.

[25] A.Y. Shen, C.P. Chen, S. Roffler, A chelating agent possessing cytotoxicity and antimicrobial activity: 7-morpholinomethyl-8-hydroxyquinoline, Life Sci. 64 (1999) 813-825.

[26] V. Prachayasittikul, S. Prachayasittikul, S. Ruchirawat, V. Prachayasittikul, 8hydroxyquinolines: a review of their metal chelating properties and medicinal applications, Drug Des. Devel. Ther. 7 (2013) 1157-1178.

[27] L. Paloque, P. Verhaeghe, M. Casanova, C. Castera-Ducros, A. Dumètre, L. Mbatchi, et al., Discovery of a new antileishmanial hit in 8-nitroquinoline series, Eur. J. Med. Chem. 54 (2012) 75-86.

[28] J.C. Coa, W. Castrillón, W. Cardona, M. Carda, V. Ospina, J.A. Muñoz, et al., Synthesis, leishmanicidal, trypanocidal and cytotoxic activity of quinoline-hydrazone hybrids, Eur. J. Med. Chem. 101 (2015) 746-753.

[29] M.C. Duarte, L.M.R. Lage, D.P. Lage, J.T. Mesquita, B.C. Salles, S.N. Lavorato, et al., An effective in vitro and in vivo antileishmanial activity and mechanism of action of 8hydroxyquinoline against Leishmania species causing visceral and tegumentary leishmaniasis, Vet. Parasitol. 217 (2016) 81-88.

[30] J.M. Barichello, M. Morishita, K. Takayama, T. Nagai, Absorption of insulin from pluronic F-127 gels following subcutaneous administration in rats, Int. J. Pharm. 184 (1999) 189-198.

[31] E.A. Coelho, C.A.P. Tavares, F.A.A. Carvalho, K.F. Chaves, K.N. Teixeira, R.C. Rodrigues, et al., Immune responses induced by the Leishmania (Leishmania) donovani A2 antigen, but not by the LACK antigen, are protective against experimental Leishmania (Leishmania) amazonensis infection, Infect. Immun. 71 (2003) 3988-3994.

[32] P.S. Doyle, J.C. Engel, P.F.P. Pimenta, P.P. Silva, D.M. Dwyer, Leishmania donovani: long-term culture of axenic amastigotes at $37^{\circ} \mathrm{C}$, Exp. Parasitol. 73 (1991) 326-334.

[33] P.S. Lage, M.A. Chávez-Fumagalli, J.T. Mesquita, L.M. Mata, S.O. Fernandes, V.N. Cardoso, et al., Antileishmanial activity and evaluation of the mechanism of action of strychnobiflavone flavonoid isolated from Strychnos pseudoquina against Leishmania infantum, Parasitol. Res. 114 (2015) 4625-4635.

[34] D.G. Valadares, M.C. Duarte, J.S. Oliveira, M.A. Chávez-Fumagalli, V.T. Martins, L.E. Costa, et al., Leishmanicidal activity of the Agaricus blazei Murill in different Leishmania species, Parasitol. Int. 60 (2011) 357-363.

[35] R.G. Titus, M. Marchand, T. Boon, J.A. Louis, A limiting dilution assay for quantifying Leishmania major in tissues of infected mice, Parasite Immunol. 7 (1985) 545-555.

[36] V.T. Martins, M.A. Chávez-Fumagalli, L.E. Costa, A.M. Canavaci, P.S. Lage, D.P. Lage, et al., Antigenicity and protective efficacy of a Leishmania amastigote-specific protein, member of the super-oxygenase family, against visceral leishmaniasis, PLoS Negl. Trop. Dis. 7 (2013), e2148.

[37] A.S. Ferreira, R.S. Leite, L.T. Ituassu, G.G. Almeida, D.M. Souza, R.T. Fujiwara, et al., Canine skin and conjunctival swab samples for the detection and quantification of Leishmania infantum DNA in an endemic urban area in Brazil, PLoS Negl. Trop. Dis. 6 (2012), e1596.

[38] D.P. Lage, V.T. Martins, M.C. Duarte, E. Garde, M.A. Chávez-Fumagalli, D. MenezesSouza, et al., Prophylactic properties of a Leishmania-specific hypothetical protein in a murine model of visceral leishmaniasis, Parasite Immunol. 37 (2015) 646-656.

[39] B.M. Roatt, R.D. Aguiar-Soares, J. Vitoriano-Souza, W. Coura-Vital, S.L. Braga, R. Corrêa-Oliveira, et al., Performance of LBSap vaccine after intradermal challenge with L. infantum and saliva of Lu. longipalpis: immunogenicity and parasitological evaluation, PLoS One 7 (2012), e49780.

[40] L.C. Green, D.A. Wagner, J. Glogowski, P.L. Skipper, J.S. Wishnok, S.R. Tannenbaum, Analysis of nitrate, nitrite, and [15N]nitrate in biological fluids, Anal. Biochem. 126 (1982) 131-138.

[41] V.T. Martins, M.C. Duarte, M.A. Chávez-Fumagalli, D. Menezes-Souza, C.S. Coelho, D.F. Magalhães-Soares, et al., A Leishmania-specific hypothetical protein expressed in both promastigote and amastigote stages of Leishmania infantum employed for the serodiagnosis of, and as a vaccine candidate against, visceral leishmaniasis, Parasitol. Vectors 8 (2015) 363.

[42] D.M. Oliveira, D.G. Valadares, M.C. Duarte, L.E. Costa, V.T. Martins, R.F. Gomes, et al., Evaluation of parasitological and immunological parameters of Leishmania chagasi infection in BALB/c mice using different doses and routes of inoculation of parasites, Parasitol. Res. 110 (2012) 1277-1285.

[43] B.L. Herwaldt, J.D. Berman, Recommendations for treating leishmaniasis with sodium stibogluconate (Pentostam) and review of pertinent clinical studies, Am.J.Trop. Med. Hyg. 46 (1992) 296-306.

[44] R.M. Fielding, P.C. Smith, L.H. Wang, J. Porter, L.S. Guo, Comparative pharmacokinetics of amphotericin $B$ after administration of a novel colloidal delivery system, ABCD, and a conventional formulation to rats, Antimicrob. Agents Chemother. 35 (1991) 1208-1213.
[45] P. Gershkovich, E.K. Wasan, M. Lin, O. Sivak, C.G. Leon, J.G. Clement, et al., Pharmacokinetics and biodistribution of amphotericin B in rats following oral administration in a novel lipid-based formulation, J. Antimicrob. Chemother. 64 (2009) 101-108.

[46] A.K. Agrawal, A. Agrawal, A. Pal, P.Y. Guru, C.M. Gupta, Superior chemotherapeutic efficacy of amphotericin B in tuftsin-bearing liposomes against Leishmania donovani infection in hamsters, J. Drug Target. 10 (2002) 41-45.

[47] S.P. Vyas, S. Gupta, Optimizing efficacy of amphotericin B through nanomodification, Int. J. Nanomedicine 1 (2006) 417-432.

[48] T.G. Ribeiro, M.A. Chávez-Fumagalli, D.G. Valadares, J.R. França, L.B. Rodrigues, M.C Duarte, et al., Novel targeting using nanoparticles: an approach to the development of an effective anti-leishmanial drug delivery system, Int. J. Nanomedicine 14 (2014) 877-890.

[49] T.G. Ribeiro, J.R. Franca, L.L. Fuscaldi, M.L. Santos, M.C. Duarte, P.S. Lage, et al., An optimized nanoparticle delivery system based on chitosan and chondroitin sulfate molecules reduces the toxicity of amphotericin B and is effective in treating tegumentary leishmaniasis, Int. J. Nanomedicine 9 (2014) 5341-5353.

[50] F. Chiellini, A.M. Piras, C. Errico, E. Chiellini, Micro/nanostructured polymeric systems for biomedical and pharmaceutical applications, Nanomedicine (London) 3 (2008) 367-393.

[51] M.S. Akash, K. Rehman, Recent progress in biomedical applications of Pluronic (PF127): pharmaceutical perspectives, J. Control. Release 209 (2015) 120-138.

[52] T. Serno, R. Geidobler, G. Winter, Protein stabilization by cyclodextrins in the liquid and dried state, Adv. Drug Deliv. Rev. 63 (2011) 1086-1106.

[53] V.P. Torchilin, T.S. Levchenko, K.R. Whiteman, A.A. Yaroslavov, A.M. Tsatsakis, A.K. Rizos, et al., Amphiphilic poly- $N$-vinylpyrrolidones: synthesis, properties and liposome surface modification, Biomaterials 22 (2001) 3035-3044.

[54] S.K. Jain, M.K. Chourasia, M. Sabitha, R. Jain, A.K. Jain, M. Ashawat, et al., Development and characterization of transdermal drug delivery systems for diltiazem hydrochloride, Drug Deliv. 10 (2003) 169-177.

[55] Y. Wang, L. Yu, L. Han, X. Sha, X. Fang, Difunctional Pluronic copolymer micelle for paclitaxel delivery: synergistic effect of folate-mediated targeting and Pluronic-mediated overcoming multidrug resistance in tumor cell lines, Int. J. Pharm. 337 (2007) 63-73.

[56] W. Zhang, Y. Shi, Y. Chen, J. Ye, X. Sha, X. Fang, Multifunctional Pluronic P123/F127 mixed polymeric micelle loaded with paclitaxel for the treatment of multidrug resistant tumors, Biomaterials 32 (2011) 2894-2906.

[57] L. Lamch, U. Bazylińska, J. Kulbacka, J. Pietkiewicz, K. Bieżuńska-Kusiak, K.A. Wilk, Polymeric micelle for enhanced Photofrin II® delivery, cytotoxicity and pro-apoptotic activity in human breast and ovarian cancer cells, Photodiagn. Photodyn. Ther. 11 (2014) 570-585

[58] R. Garg, A. Dube, Animal models for vaccine studies for visceral leishmaniasis, Indian J. Med. Res. 123 (2006) 439-454.

[59] A.P. Brugués, B.C. Naveros, A.C. Campmany, P.H. Pastor, R.F. Saladrigas, C.R. Lizandra, Developing cutaneous applications of pamamomycin entrapped in stimuli-sensitive block copolymer nanogeldispersions, Nanomedicine (London) 10 (2015) 227-240.

[60] P.S. Lage, P.H.R. Andrade, A.D.S. Lopes, M.A. Chávez-Fumagalli, D.G. Valadares, M.C. Duarte, et al., Strychnos pseudoquina and its purified compounds present an effective in vitro antileishmanial activity, Evid. Based Complement. Altern. Med. 1 (2013) 9.

[61] T.G. Ribeiro, A.M. Nascimento, B.O. Henriques, M.A. Chávez-Fumagalli, J.R. Franca, M.C. Duarte, et al., Antileishmanial activity of standardized fractions of Stryphnodendron obovatum (Barbatimão) extract and constituent compounds, J. Ethnopharmacol. 165 (2015) 238-242.

[62] D.G. Valadares, M.C. Duarte, L. Ramírez, M.A. Chávez-Fumagalli, V.T. Martins, L.E Costa, et al., Prophylactic or therapeutic administration of Agaricus blazei Murill is effective in treatment of murine visceral leishmaniasis, Exp. Parasitol. 132 (2012) $228-236$.

[63] J. Carrión, A. Nieto, S. Iborra, V. Iniesta, M. Soto, C. Folgueira, et al. Immunohistological features of visceral leishmaniasis in BALB/c mice, Parasite Immunol. 28 (2006) 173-183.

[64] P.C. Melby, Y.Z. Yang, J. Cheng, W. Zhao, Regional differences in the cellular immune response to experimental cutaneous or visceral infection with Leishmania donovani, Infect. Immun. 66 (1998) 18-27.

[65] C.R. Engwerda, P.M. Kaye, Organ-specific immune responses associated with infectious disease, Immunol. Today 21 (2000) 73-78.

[66] S. Ahmed, M. Colmenares, L. Soong, K. Goldsmith-Pestana, L. Munstermann, R. Molina, et al., Intradermal infection model for pathogenesis and vaccine studies of murine visceral leishmaniasis, Infect. Immunol. 71 (2003) 401-410.

[67] A.G. Tempone, H.F. Andrade, P.J. Spencer, C.O. Lourenço, J.R. Rogero, N. Nascimento, Bothrops moojeni venom kills Leishmania spp. with hydrogen peroxide generated by its L-amino acid oxidase, Biochem. Biophys. Res. Commun. 280 (2001) 620-624.

[68] P.S. Lage, M.A. Chávez-Fumagalli, J.T. Mesquita, L.M. Mata, S.O. Fernandes, V.N Cardoso, et al., Antileishmanial activity and evaluation of the mechanism of action of strychnobiflavone flavonoid isolated from Strychnos pseudoquina against Leishmania infantum, Parasitol. Res. 114 (2015) 4625-4635.

[69] W.H. Beggs, Physicochemical cell damage in relation to lethal amphotericin B action, Antimicrob. Agents Chemother. 38 (1994) 363-364.

[70] J. Bolard, A. Vertut-Doi, Internalisation of amphotericin B and other polyene antifungals in mammalian cells: a possible origin of their toxicity, in: N.H. Georgopapdakou (Ed.), Drug Transport in Antimicrobial and Anticancer Chemotherapy, Marcel Dekker, NY 1995, p. 307.

[71] V. Oliveri, G. Vecchio, 8-Hydroxyquinolines in medicinal chemistry: a structural perspective, Eur. J. Med. Chem. 120 (2016) 252-274. 